6. Huang WT, Wang CC, Chen WJ, Cheng YF, Eng HL. The nodular form of hepatic tuberculosis: a review with five additional new cases. J Clin Pathol. 2003;56:835-9.

7. Yu RS, Zhang SZ, Wu JJ, Li RF. Imaging diagnosis of 12 patients with hepatic tuberculosis. World $J$ Gastroenterol. 2004;10:1639-42.

8. Diaz ML, Herrera T, Lopez-Vidal Y, Calva JJ, Hernandez R, Palacios GR, et al. Polymerase chain reaction for the detection of Mycobacterium tuberculosis DNA in tissue and assessment of its utility in the diagnosis of hepatic granulomas. J Lab Clin Med. 1996;127:359-63.

\title{
Lymphoepithelioma-like carcinoma: an unusual gall bladder tumour
}

\section{Introduction}

Lymphoepithelioma-like carcinoma (LLC) of the biliary tract is a relatively recently recognized entity. LLC involving the gall bladder (LLC-GB) has been described only once in the literature. ${ }^{1}$

\section{Case report}

A 64-year-old lady presented with right upper abdominal pain of three months duration. Clinical examination was normal. CT scan (Figure 1) revealed a $2.4 \times 2.0 \times 3.1 \mathrm{~cm}$ heterogeneously enhancing soft tissue lesion arising in relation to the gall bladder neck. The common bile duct was dilated $(10 \mathrm{~mm})$ till its lower end with no evidence of obstruction at the ampulla. Subcentimetric lymph nodes were present in the hepatoduodenal ligament. A provisional diagnosis of carcinoma gall bladder was made and the patient was taken up for surgery. At surgery, there was a $3 \mathrm{~cm}$ mass arising from the gallbladder neck and confined to the gall bladder. There were no peritoneal or liver metastases. Extended cholecystectomy was performed. Cystic duct margin was free of tumor on frozen section analysis. The postoperative course was uneventful and the patient was discharged in satisfactory condition. Histopathology of the resected specimen showed surface epithelial ulceration and infiltration by a tumor composed of syncytial sheets and islands of atypical polygonal cells intermixed and surrounded by

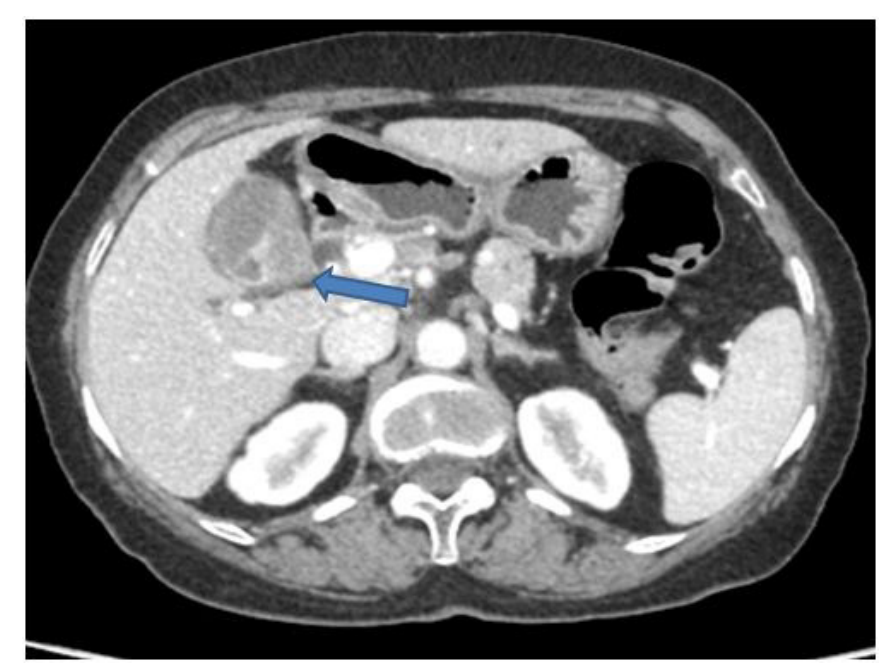

Figure 1: CECT showing mass in the neck of gall bladder (arrow)

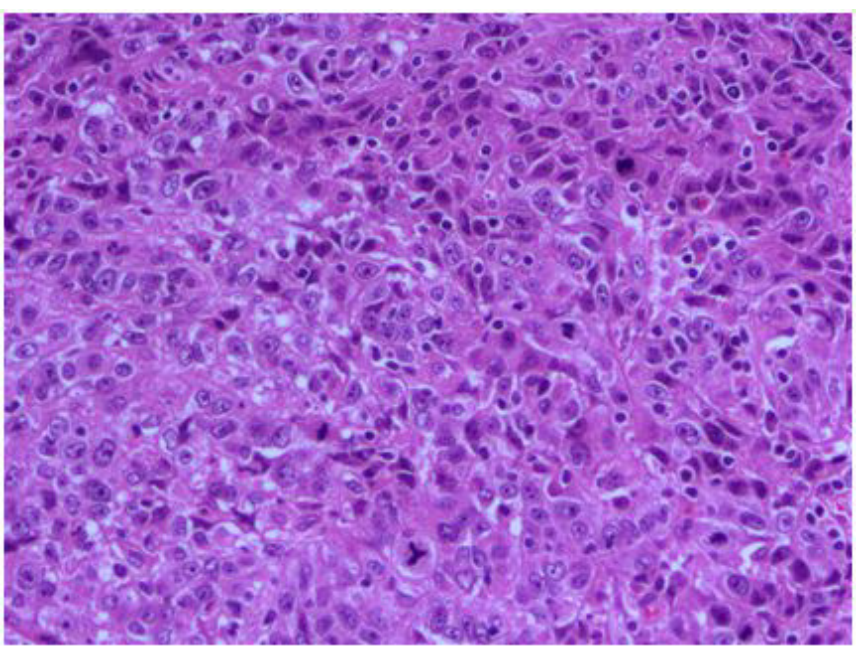

Figure 2: Tumor histopathology showing syncytial sheets and islands of atypical polygonal cells intermixed and surrounded by benign lymphoid cells (haematoxylin and eosin, 100x)

lymphoid cells (Figure 2). The tumor had infiltrated the mucosa, muscularis propria and had reached up to the subserosa. Perineural invasion was seen but there was no lymphovascular invasion. Cystic duct margin, liver transection margin and all resected lymph nodes were free of tumour. Tumour cells showed positive cytoplasmic staining with pancytokeratin and were for negative for Epstein-Barr virus on immunohistochemistry. Patient has been followed up for 18 months after surgery with no evidence of recurrence.

\section{Discussion}

Schmincke in 1921 described a distinct type of undifferentiated nasopharyngeal carcinoma where tumor cells grow in a diffuse manner mimicking a malignant lymphoma. ${ }^{2}$ This tumor has been designated as lymphoepithelial carcinoma or undifferentiated 
carcinoma with lymphocytic stroma. Several studies have reported the presence of similar tumor in several extrapharyngeal sites such as lungs, stomach, salivary glands and thymus. ${ }^{3}$ There are multiple recent case reports describing lymphoepithelioma-like carcinoma (LLC) of the biliary tract with intra-hepatic bile duct being the most common site of origin. ${ }^{2}$ To the best of our knowledge, only two cases of extrahepatic LLC have been reported, one in the gall bladder and another in the distal bile duct. ${ }^{1,4}$ Extra-nasopharyngeal LLC are also associated with EBV infection. ${ }^{2}$ The prognosis of biliary tract LLC is generally considered better than adenocarcinoma. ${ }^{5}$ This case has been reported for its rarity and lack of association with EBV infection.

PIYUSH KUMAR SINHA ${ }^{1}$ VIVEK MANGLA ${ }^{1}$ CHHAGAN BEHARI ${ }^{2}$ ARCHANA RASTOGI ${ }^{2}$ TK CHATTOPDHYAY ${ }^{1}$

Correspondence: Dr. Vivek Mangla Departments of HPB Surgery ${ }^{l}$ and Pathology, ${ }^{2}$ Institute of Liver and Biliary Sciences, D1, Vasant Kunj, New Delhi - 110070, India Email: mangla.vivek@gmail.com

\section{References}

1. Todd DL, Ro JY, Gully LM, Ayala AG. Lymphoepitheliomalike carcinoma of the gallbladder. Int J Surg Pathol. 1996;4:183-7.

2. Schmincke A. Uber lymphoepitheliale geschwulste. Beitr pathol. Anat Allg Pathol. 1921;68:161-70.

3. Iezzoni JC, Gaffey MJ, Weiss LM. The role of Epstein-Barr virus in lymphoepithelioma-like carcinomas. Am J Clin Pathol. 1995;103:308-15.

4. Ishida M, Mori T, Shiomi H, Naka S, Tsujikawa T, Andoh A, et al. Non-Epstein-Barr virus associated lymphoepithelioma-like carcinoma of the inferior common bile duct. World J Gastrointest Oncol. 2011;3:111-5.

5. Henderson-Jackson E, Nasir NA, Hakam A, Nasir A, Coppola D. Primary mixed lymphoepithelioma-like carcinoma and intrahepatic cholangiocarcinoma: a case report and review of literature. Int J Clin Exp Pathol. 2010;3:736-41.

\section{Introduction}

Visceral artery aneurysm (VAA) is often asymptomatic and discovered incidentally. Reports on ileocolic artery aneurysms (ICAA) are rare and mostly anecdotal. Therapeutic options include surgery or radiology-guided endovascular measures. We report a case of spontaneous ICAA where N-butyl cyanoacrylate (NBCA) glue was used to achieve embolisation percutaneously under ultrasound guidance.

\section{Case report}

An 81-year-old gentleman presented with two weeks history of lower abdominal pain. The pain was dull aching, continuous with no radiation or aggravation by food. There were no associated systemic symptoms like fever, loss of appetite or weight loss. Bowel and bladder habits were unaltered. He had chronic obstructive pulmonary disease and Parkinson's disease. He was treated successfully by Heller's cardiomyotomy for achalasia in 2008, with difficult endotracheal intubation prior to anaesthesia.

On examination, the patient was afebrile with other vital parameters being normal. On deep palpation tenderness was noted in the hypogastrium. There were no signs of gut obstruction or any palpable mass. Hemogram, urine examination, renal and liver function tests were normal. Ultrasound abdomen with Doppler (Figure 1A) showed a $3 \times 3$ $\mathrm{cm}$ pulsatile aneurysm. The origin of the aneurysm was unclear. The abdominal aorta, its bifurcation, the celiac and superior mesenteric arteries at their origins were all normal with no evidence of thrombosis. CT angiography (Figure 2) performed to identify the origin of the aneurysm, showed a $4.5 \times 4 \mathrm{~cm}$ aneurysm filled with a partial thrombus originating from the ileocolic branch of superior mesenteric artery (SMA), with no other aneurysms elsewhere in the abdomen. The afferent artery was $2 \mathrm{~mm}$ in size. $2 \mathrm{ml} \mathrm{NBCA}$ was injected into the aneurysm percutaneously under ultrasound guidance with no complications. The patient improved symptomatically within 24 hours post-procedure. At 4 weeks follow-up the patient was asymptomatic and Doppler ultrasound showed complete embolisation of the aneurysm (Figure 1B).

\section{Discussion}

\section{Ultrasound-guided glue injection of ileocolic artery aneurysm}

VAA are intra-abdominal aneurysms that affect the celiac, superior and inferior mesenteric, renal arteries and their 